\title{
IFAR Liner Benchmark - Challenge \#1 - DLR
}

\author{
Friedrich Bake*, Ralf Burgmayer; Anita Schulz $z^{\ddagger}$ and Lars Enghardt ${ }^{\S}$ \\ German Aerospace Center (DLR), Institute of Propulsion Technology, Engine Acoustics, D-10623 Berlin, Germany
}

\begin{abstract}
This paper presents the contribution from the German Aerospace Center (DLR) to the first challenge of a liner benchmark under the framework of the International Forum for Aviation Research (IFAR). Therefore, two sets of acoustically damping wall treatment, called liner samples, have been produced by additive manufacturing based on the design data provided by NASA coordinating this benchmark. These liner samples have been integrated and acoustically characterized in the liner flow test facility DUCT-R at DLR Berlin. Besides the dissipation coefficients and the axial pressure profiles the liner wall impedance was educed by first determining the axial wave numbers and then applying a straightforward method based on the one-dimensional Convected Helmholtz Equation.
\end{abstract}

\section{Introduction}

Acoustic liners as a wall treatment are one of the most important technology for aircraft noise reduction. Liners are especially integrated in the inlet and bypass duct of aero-engines in order to reduce the engine noise emission. Although there are existing fairly mature technology solutions for liner design, the prediction abilities of liner performance under real application conditions are still unsatisfactory. There remains the need for experimental testing of liner samples in purpose built liner flow duct facilities. This yields the challenge to ensure comparability between the different worldwide existing liner test facilities and the correspondingly applied post-processing methods and prediction approaches.

Therefore, the International Forum for Aviation Research (IFAR) initiated a benchmark activity devoted to compare acoustic liner testing and performance prediction. The DLR Department of Engine Acoustics takes part in this liner benchmark managed by NASA. The liner benchmark is divided into three different challenges:

- Challenge \#1: Comparison of Liner Test Facilities

- Challenge \#2: Propagation Code Comparison

- Challenge \#3: Impedance Eduction Comparison

This paper will mainly focus on the DLR contribution to challenge \#1. Hereby, NASA provided CAD drawing data of selected generic liner designs for all participating partners within IFAR. Two liner configurations are considered for this challenge. The first is a uniform liner, for which the impedance should be nearly constant over the length of the liner. The second is a two-segment liner, where the only difference between the two axial segments is the depth of the liner cavity. First measurement results from these liner configurations have been published by NASA in Jones et al. ${ }^{1}$.

The goal of challenge \#1 is to gather data from multiple test rigs with the same liner configurations manufactured using 3D printing and to share data with each participant to evaluate dependence of our results on fabrication, data acquisition and analysis (e.g., impedance eduction) approaches.

Within this paper the DLR geometric liner adaptation, the liner manufacturing process, the test facility and measurement procedure and the final acoustic results will be presented.

\footnotetext{
* Scientist, DLR Institute of Propulsion Technology, Engine Acoustics, AIAA Member. Friedrich.Bake@dlr.de.

${ }^{\dagger}$ Research Associate, DLR Institute of Propulsion Technology, Engine Acoustics. Ralf.Burmayer@dlr.de.

$\ddagger$ Postdoc, DLR Institute of Propulsion Technology, Engine Acoustics. Anita.Schulz@dlr.de.

$\S$ Professor, DLR Institute of Propulsion Technology, Engine Acoustics, AIAA Member. Lars.Enghardt@dlr.de.
} 


\section{Liner Manufacturing}

Figure 1 shows the liner configurations under test. The first, homogeneous liner sample IFAR1 has a constant cavity depth of $2.0^{\prime \prime}$ and the second liner sample IFAR2 has one part with a cavity depth of also $2.0^{\prime \prime}$ and another part with a cavity depth of $3.0^{\prime \prime}$. Both liner samples consist of 4-by-30 liner cavities. The liner cavity dimensions are $0.4^{\prime \prime}$ by $0.4^{\prime \prime}$ with an axial partition thickness of $0.133^{\prime \prime}$ and a spanwise partition thickness of $0.129^{\prime \prime}$ giving a total length of $16^{\prime \prime}$ and a total width of $2.265^{\prime \prime}$. Each liner cavity has 22 holes with a nominal diameter of $0.044^{\prime \prime}$, yielding a porosity of about $12 \%$, including partition thicknesses. The face sheet has a thickness of $0.032^{\prime \prime}$.

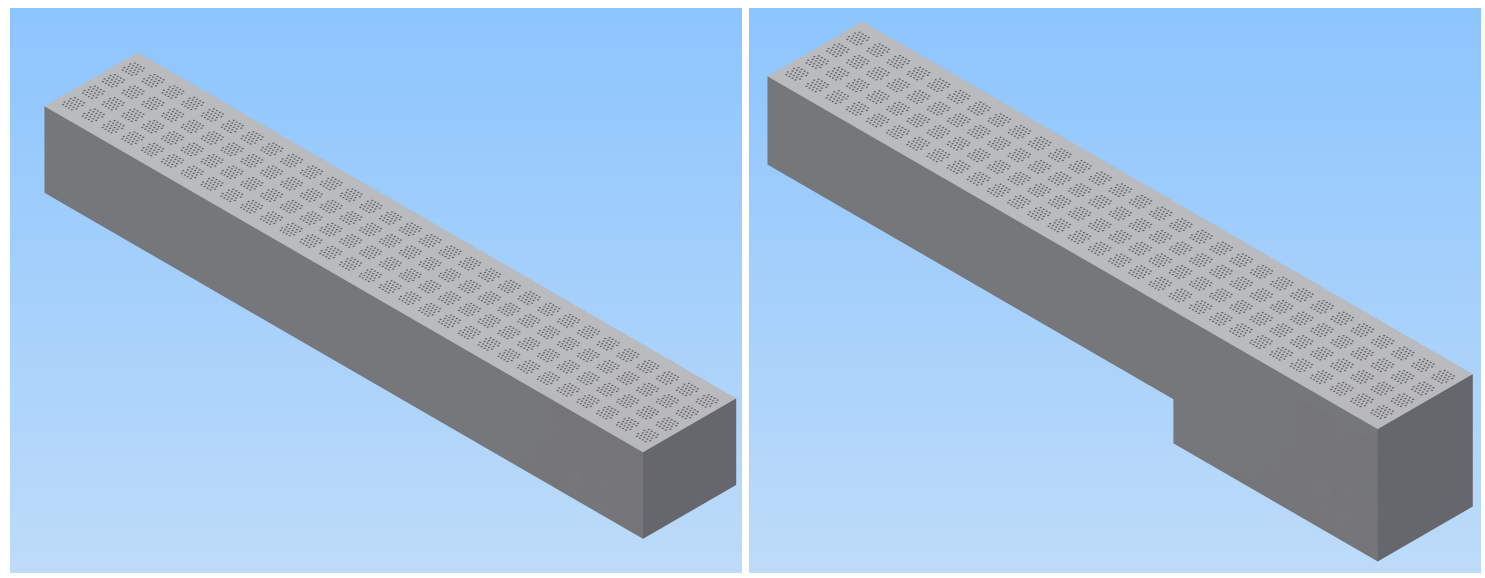

Figure 1. CAD models of the liner samples (left: constant depth liner IFAR1; right: two-segment liner IFAR2).

To fit into the liner mounting device of the DLR liner test facility DUCT-R, the provided CAD data was adapted with a minor modification, increasing the thickness of outer axial partitions to $0.246^{\prime \prime}$. For the manufacturing process the liner samples were 3D-printed using stereolithography indurating liquid photopolymer resin. Since the length of the liner samples exceeds the maximum printable size of our device, the samples were printed in three segments consisting each of 4-by-10 cavities. As a backplate, a $0.149^{\prime \prime}$ thick aluminum plate was used.

Because the size of the liner segments corresponds to almost the maximum permissible printing size of our device, consequentially resulting in inaccurate prints, the main challenge during manufacturing was to find the best suiting printing configuration to obtain optimal printing accuracy. For this purpose, a number of test prints with varying print parameters were conducted. To further increase manufacturing accuracy, all samples were designed with a $0.04^{\prime \prime}$ oversize. During post processing the samples were sanded and the oversize was milled to match the nominal specifications. Subsequently the segments were evaluated yielding maximum deviations of about $0.002^{\prime \prime}$ compared to the required specifications. The perforation diameters vary between $0.042^{\prime \prime}$ and $0.044^{\prime \prime}$. The left photo in figure 2 shows a single liner segment after post processing and the right photo shows the homogeneous liner sample installed in the DUCT-R.
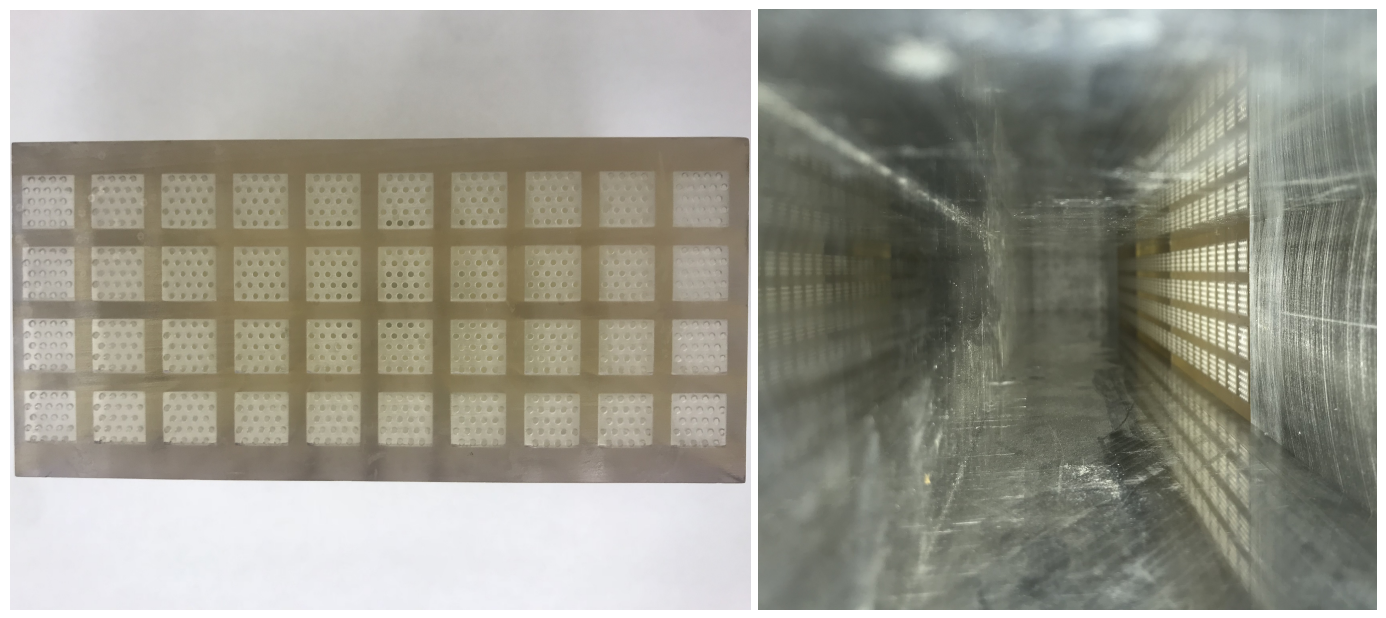

Figure 2. Photos of the 3D printed post processed liners (left: single liner segment; right: liner sample installed in the duct). 


\section{Liner Test Facility and Measurement Setup}

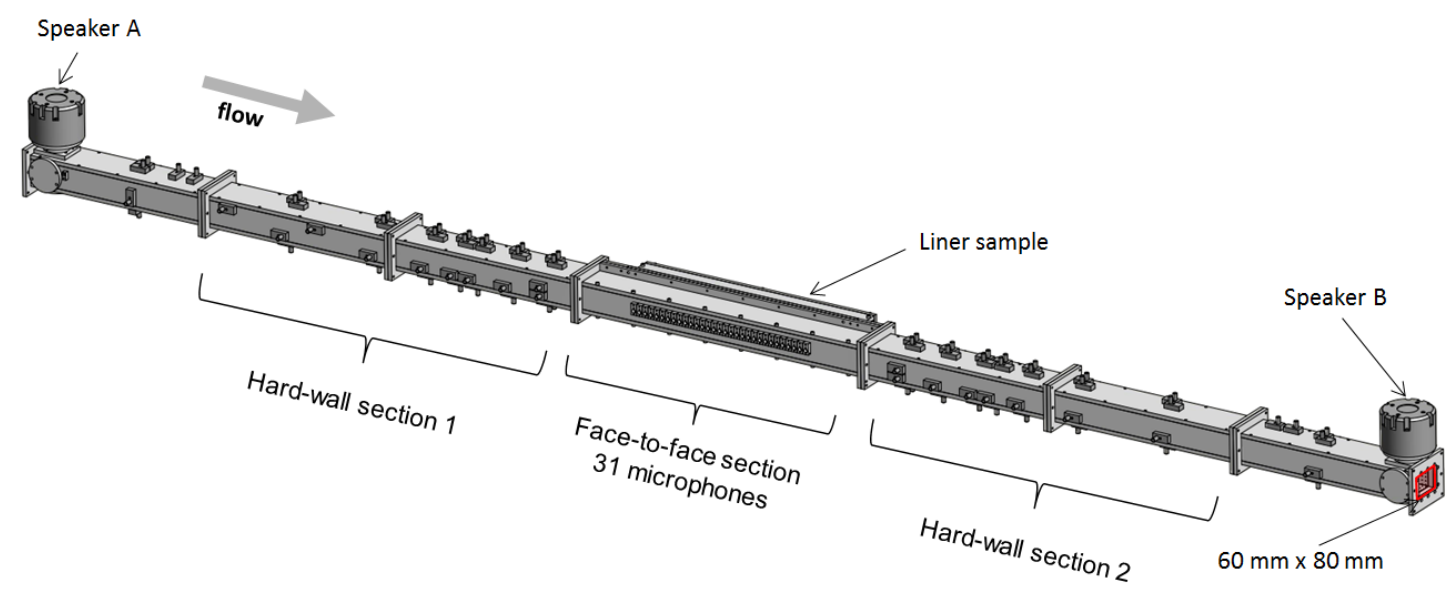

Figure 3. Sketch of the DLR liner test facility DUCT-R with the two measurement (hard-wall) sections and the liner mounting module in between.

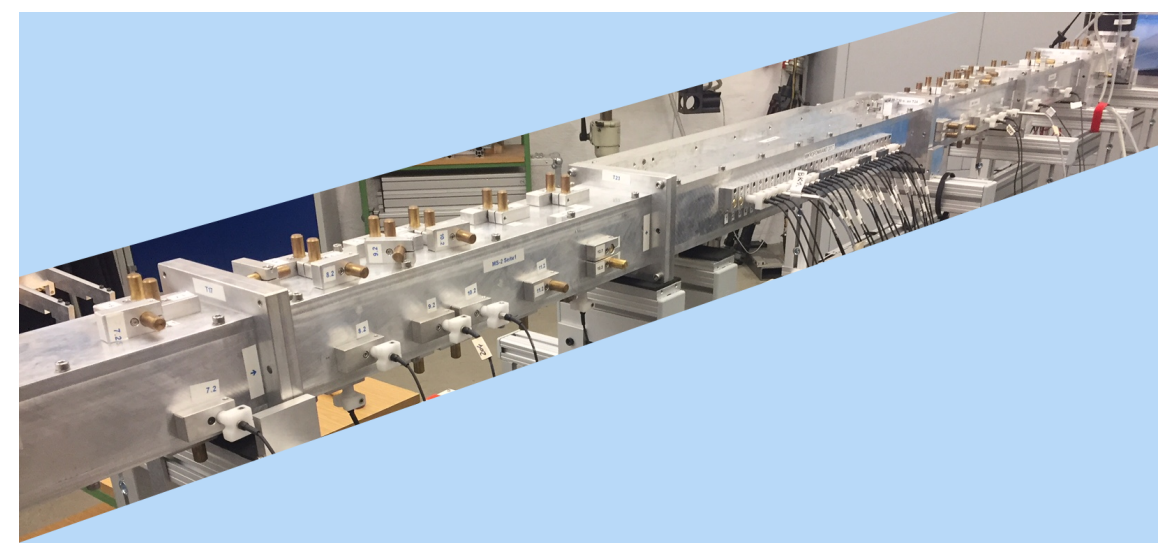

Figure 4. Photo of the DLR liner test facility DUCT-R with the liner mounting module in the center.

The DLR liner test facility DUCT-R consists of a flow duct with a cross-section of $60 \mathrm{~mm}(W)$ by $80 \mathrm{~mm}(H)$. Driven by an upstream radial compressor a mean flow Mach number on the centerline of up to 0.3 can be set. The duct itself is setup symmetrically with loudspeakers (A and B) at the upstream and downstream end, two (hard-wall) measurement sections and a liner mounting module (face-to-face section) in the center as shown in the schematic sketch in figure 3. Microphones are installed at different axial positions in both the hard wall measurement sections as well as face-to-face in the liner module on the opposite site of the liner surface. Figure 4 shows a photo of the DUCT-R setup applied for this investigation. Further details about the test rig and the acoustic measurements system can also be found in Busse-Gerstengarbe et al. ${ }^{2}$ and in Schulz et al. ${ }^{3}$ The variety of different microphone positions enables the evaluation of the scattering coefficients (reflection, transmission and dissipation) of the liner module via the hard-wall measurement sections and the acquisition of the sound field directly in the lined duct section via the face-to-face microphones. From both data sets a determination of the liner impedance via different eduction methods is possible. This includes modal and non-modal based impedance eduction approaches as described in Weng et al. ${ }^{4}$

\section{Data Post-Processing and Analysis}

This section presents the data analysis steps and the corresponding results from the liner samples IFAR1 and IFAR2. 


\section{IV.A. Scattering Coefficients}

The damping performance of the liner is evaluated using the dissipation coefficient. This is an integral value of the acoustic power that is absorbed while a sound wave is passing the lined element. The determination of the dissipation coefficient like also documented by Lahiri et al. ${ }^{5}$ is based on a method proposed by Ronneberger ${ }^{6}$ and his students. ${ }^{7,8}$ For each configuration two different sound fields are excited consecutively in two separate measurements (index a and b). Speaker A (upstream) is used in the first measurement and in the second measurement the same signal is fed into speaker B (downstream). Then, the data of section 1 and section 2 (index 1 and 2) are analyzed separately. This results in four equations for the complex sound pressure amplitudes for each section and measurement:

$$
\begin{aligned}
& p_{1 a}(x)=p_{1 a}^{+} e^{-i k_{1}^{+} x}+p_{1 a}^{-} e^{i k_{1}^{-} x} \\
& p_{2 a}(x)=p_{2 a}^{+} e^{-i k_{2}^{+} x}+p_{2 a}^{-} e^{i k_{2}^{-} x} \\
& p_{1 b}(x)=p_{1 b}^{+} e^{-i k_{1}^{+} x}+p_{1 b}^{-} e^{i k_{1}^{-} x} \\
& p_{2 b}(x)=p_{2 b}^{+} e^{-i k_{2}^{+} x}+p_{2 b}^{-} e^{i k_{2}^{-} x}
\end{aligned}
$$

$p^{+}$and $p^{-}$are the complex amplitudes of the downstream and upstream traveling waves with their respective wave numbers $k^{ \pm}$. The amplitude of the incident sound wave was set to approx. $110 \mathrm{~dB}$.

The recorded microphone signals are transformed into the frequency domain using the method presented by Chung. ${ }^{9}$ This method rejects uncorrelated noise, e.g. turbulent flow noise, from the coherent sound pressure signals. Therefore, the sound pressure spectrum of one microphone is determined by calculating the cross-spectral densities between three signals, where one signal serves as a phase reference. In our case the phase reference signal is the source signal of the active loudspeaker. As a result we obtain a phase-correlated complex sound pressure spectrum for each microphone signal.

According to Eqs. 1-4 the measured acoustic signal is a superposition of two plane waves traveling in opposite direction. In order to determine the downstream and upstream propagating portions of the wave in each section, Eqs. 1-4 are fitted to the microphone data. As a result of this least-mean-square fit, the four complex sound pressure amplitudes $p_{1}^{+}, p_{1}^{-}, p_{2}^{+}$and $p_{2}^{-}$are identified for both measurements. The respective ratio of these sound pressure amplitudes yield the reflection and transmission coefficients of the test object. This is illustrated in Fig. 5 for the two different measurements $A$ and $B$. The results from both measurements are combined in order to calculate the reflection and transmission coefficients:

$$
\begin{gathered}
r^{+}=\frac{p_{1 a}^{-} p_{2 b}^{-}-p_{1 b}^{-} p_{2 a}^{-}}{p_{1 a}^{+} p_{2 b}^{-}-p_{1 b}^{+} p_{2 a}^{-}} \quad r^{-}=\frac{p_{2 b}^{+} p_{1 a}^{+}-p_{2 a}^{+} p_{1 b}^{+}}{p_{1 a}^{+} p_{2 b}^{-}-p_{1 b}^{+} p_{2 a}^{-}} \\
t^{+}=\frac{p_{2 a}^{+} p_{2 b}^{-}-p_{2 b}^{+} p_{2 a}^{-}}{p_{1 a}^{+} p_{2 b}^{-}-p_{1 b}^{+} p_{2 a}^{-}} \quad t^{-}=\frac{p_{1 a}^{+} p_{1 b}^{-}-p_{1 b}^{+} p_{1 a}^{-}}{p_{1 a}^{+} p_{2 b}^{-}-p_{1 b}^{+} p_{2 a}^{-}}
\end{gathered}
$$

The advantage of combining the two measurements is that the resulting coefficients are independent from the reflection of sound at the duct terminations. These end-reflections are contained in the equations of the sound pressure amplitudes, but do not need to be calculated explicitly. The analysis is applied only in the plane-wave regime (here up to $2100 \mathrm{~Hz}$ ) i.e. the acoustic pressure is constant across the duct cross-section.

The dissipation coefficient of the acoustic energy can be calculated from the reflection and transmission coefficients via an energy balance:

$$
R^{ \pm}+T^{ \pm}+\Delta^{ \pm}=1
$$

The energy of the incident wave is partly reflected, partly transmitted, and partly absorbed by the damping module. $R$ and $T$ are the power quantities of the reflection and transmission coefficients, while $r$ and $t$ have been the pressure quantities. Blokhintsev ${ }^{10}$ defines the acoustic energy flux $I$ in a moving medium (see as well in $^{11}$ ):

$$
I=\frac{1}{\rho c}(1+M)^{2}\left\langle p^{2}\right\rangle
$$

where $\langle p\rangle$ is the time-averaged acoustic pressure, $\rho$ is the density of the medium, $c$ is the speed of sound, and $M$ is the mean Mach number. Integrating over the duct cross-section area $A$ and using the pressure amplitude yields a relation between the acoustic pressure $p$ and acoustic power $P$ quantities:

$$
P^{ \pm}=\frac{A}{2 \rho c}(1 \pm M)^{2}\left|p^{ \pm}\right|^{2}
$$




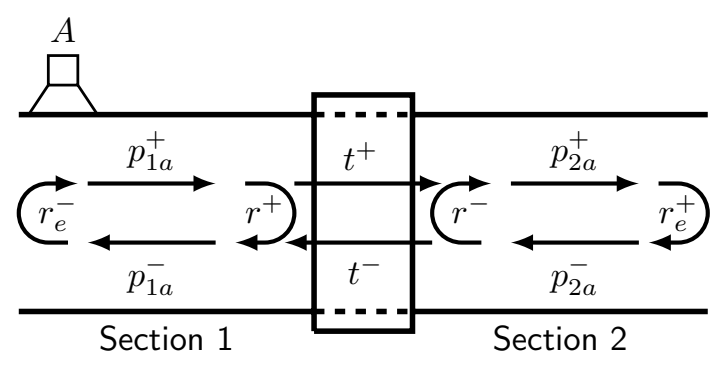

(a) Measurement A, upstream excitation

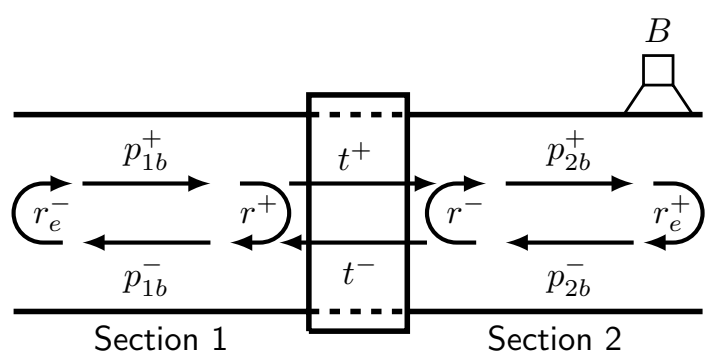

(b) Measurement B, upstream excitation

Figure 5. Illustration of the sound field in the duct for measurements $A$ and $B$ by means of the sound pressure amplitudes $p$, the reflection coefficient $r$, the transmission coefficient $t$, and the end reflection $r_{e}$

Applying Eq. 9 to $R$ and $T$ in Eq. 7 and then solving for $\Delta$ with $A_{1}=A_{2}, \rho_{1}=\rho_{2}, c_{1}=c_{2}$, and $M_{1}=M_{2}=M$ yields the definition of the energy dissipation coefficient:

$$
\Delta^{ \pm}=1-\left(\frac{(1 \mp M)^{2}}{(1 \pm M)^{2}} \cdot\left|r^{ \pm}\right|^{2}+\left|t^{ \pm}\right|^{2}\right)
$$

This is an integral value of the acoustic energy that is absorbed while a sound wave is passing the damping module.
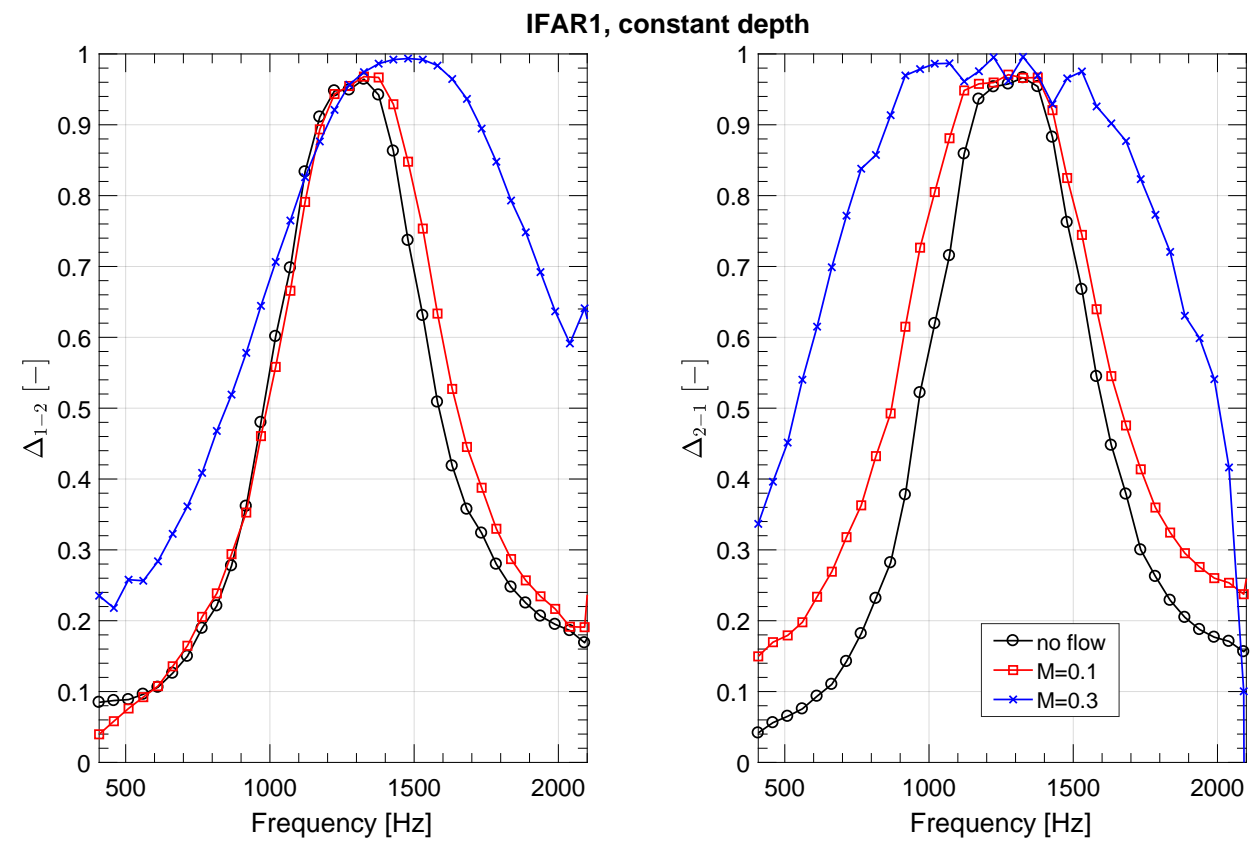

Figure 6. Dissipation coefficient of IFAR1 liner sample for different flow conditions: no flow (blue), centerline Mach number 0.1 (red) and centerline Mach number 0.3 (black). left: upstream excitation; right: downstream excitation.

Figure 6 presents the dissipation coefficient of the IFAR1 liner sample with the constant cavity depth for the three investigated flow cases:

- no flow

- centerline Mach number: 0.1

- centerline Mach number: 0.3

The left side of figure 6 shows the dissipation coefficient according to an incident wave from the upstream direction which is in the no flow case (black line) fairly identical to the one according to an incident wave from the downstream 
direction (right side of figure 6) since the liner sample is symmetric. It provides a resonator liner typical, distinct dissipation maximum here at around $1300 \mathrm{~Hz}$ which broadens with increased grazing flow Mach number. This frequency is presumably related to a quarter-wavelength $(\lambda / 4)$ resonance in the liner cavity. For a grazing flow Mach number of 0.3 the dissipation maximum for upstream excitation shifts slightly to higher frequencies around $1500 \mathrm{~Hz}$. In the opposite direction against the flow the broadening of the high dissipation range is stronger as expected. In this case the transmission coefficient (not shown here) is de facto zero for a frequency range between $1100 \mathrm{~Hz}$ and $1400 \mathrm{~Hz}$.
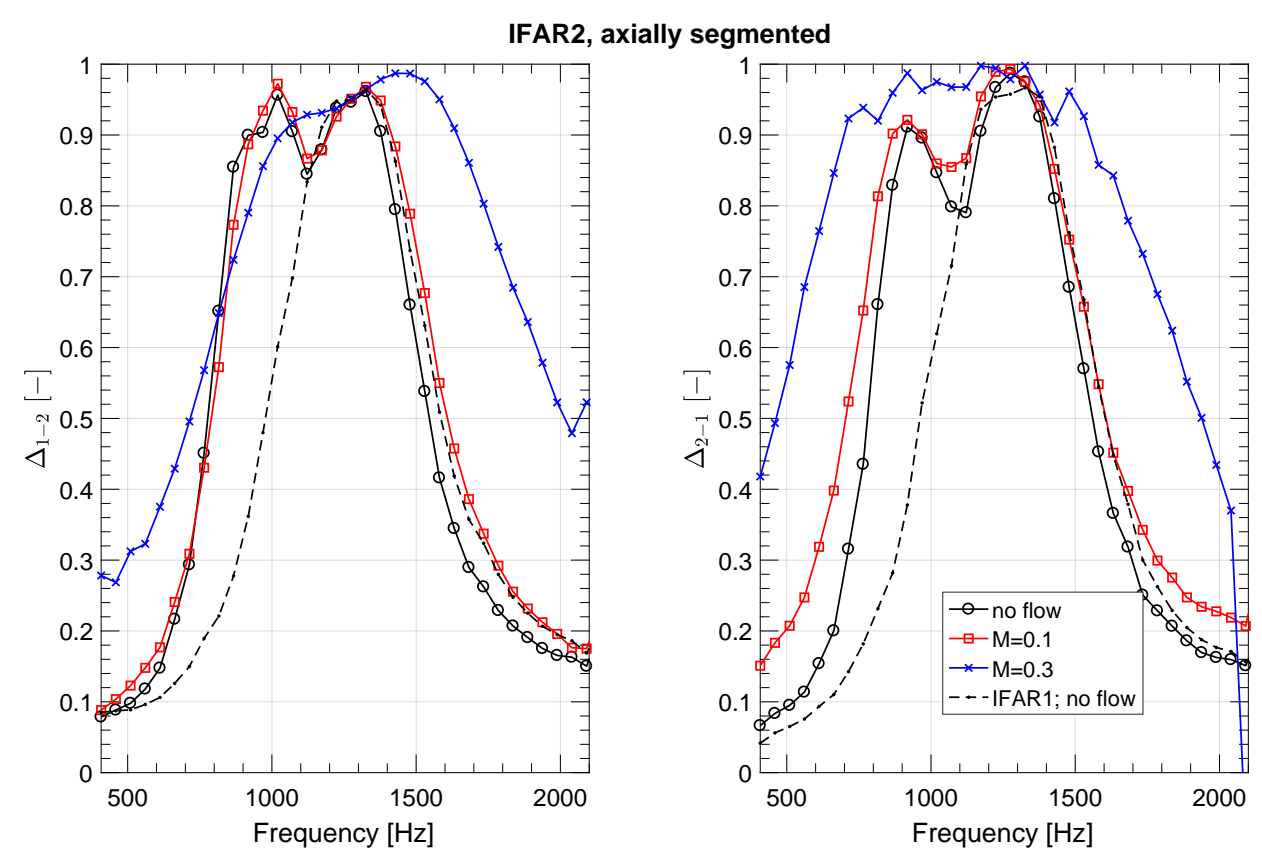

Figure 7. Dissipation coefficient of IFAR2 liner sample for different flow conditions: no flow (blue), centerline Mach number 0.1 (red) and centerline Mach number 0.3 (black). left: upstream excitation; right: downstream excitation. The black dashed line shows the no flow results of the IFAR1 sample (constant depth).

The dissipation coefficient of the axially segmented IFAR2 liner in figure 7 exhibits for the no flow case (black solid line) two maxima at around $1100 \mathrm{~Hz}$ and again at around $1300 \mathrm{~Hz}$. The dissipation coefficient of the IFAR1 liner sample (black dashed line) which has the same geometrical configuration ( $2^{\prime \prime}$ cavity depth) than the first segment of IFAR2 masks fairly well the second dissipation maximum. The first dissipation maximum seems to be related to the second axial liner segment with a cavity depth of $3^{\prime \prime}$. With increasing grazing flow Mach number (red and blue line) a similar broadening effect of the dissipation is observable like for the IFAR1 liner. At a Mach number of 0.3 the two dissipation maxima almost merge.

\section{IV.B. Axial Pressure Profiles}

In order to enhance the understanding of the damping characteristics and to prepare the wave number determination in the lined duct sections, figures 8 (IFAR1) and 9 (IFAR2) show the axial pressure profile for selected frequencies of the face-to-face mounted microphones in the liner section with the two closest microphones on each side in the hard wall sections. Both figures display the case with upstream excitation (speaker A) and a grazing flow Mach number of $M=0.3$. The axial position and the length of the lined section is marked with a gray box.

The magnitude of the sound pressure for the IFAR1 liner sample (constant depth) in figure 8 is strongly decreasing over the lined section for the center frequency of the dissipation maximum $(1479 \mathrm{~Hz}$, red line). For the frequency, where the dissipation is significantly lower $(765 \mathrm{~Hz}$, blue line $)$, the decline in the axial pressure profile is smaller while a standing wave pattern in the lined section due to reflections becomes more prominent.

The IFAR2 liner with axial segmentation in figure 9 provides two different frequencies with significant sound pressure reduction corresponding to the two dissipation peaks (see figure 7). The first frequency of $1122 \mathrm{~Hz}$ (red line) shows a sound pressure reduction above the second liner segment ( $3^{\prime \prime}$ cavity depth) at $0.467 \mathrm{~m}<x<0.603 \mathrm{~m}$. Whereas for the second frequency of $1479 \mathrm{~Hz}$ (green line) the sound pressure is mainly reduced in the liner segment with the lower cavity depth of $2^{\prime \prime}$ comparable to the IFAR1 sample with the same cavity depth. The pressure profile of 


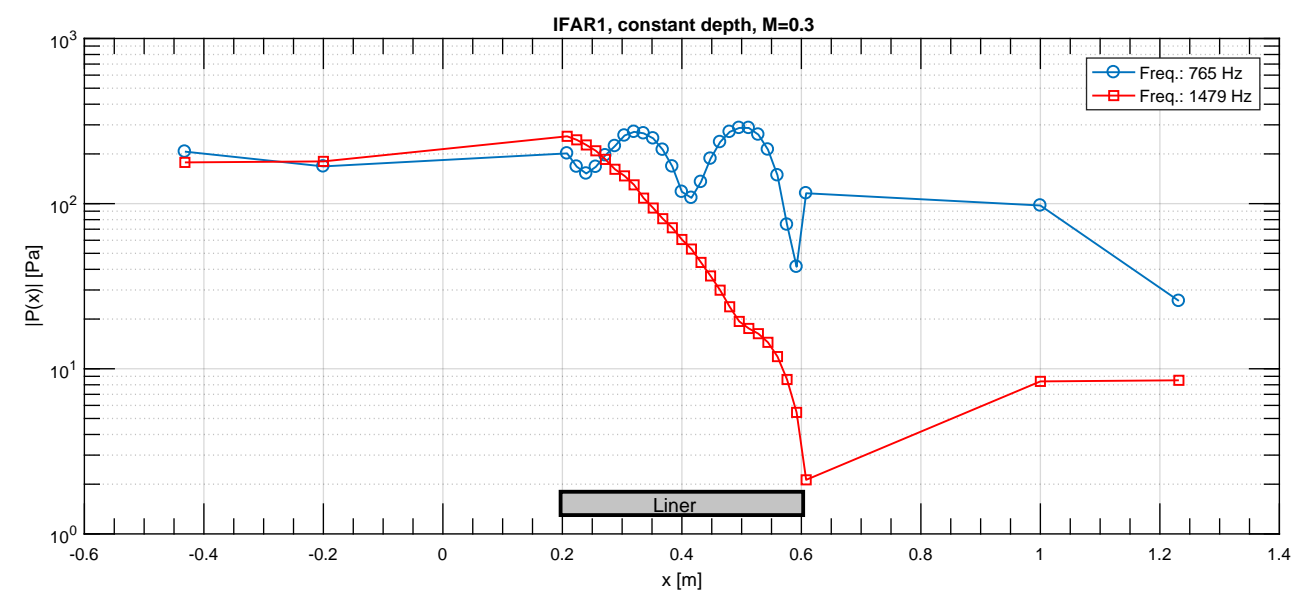

Figure 8. Axial pressure profiles with IFAR1 liner sample at centerline Mach number 0.3 for two different frequencies: $867 \mathrm{~Hz}$ (blue line; low dissipation) and $1326 \mathrm{~Hz}$ (red line; high dissipation).

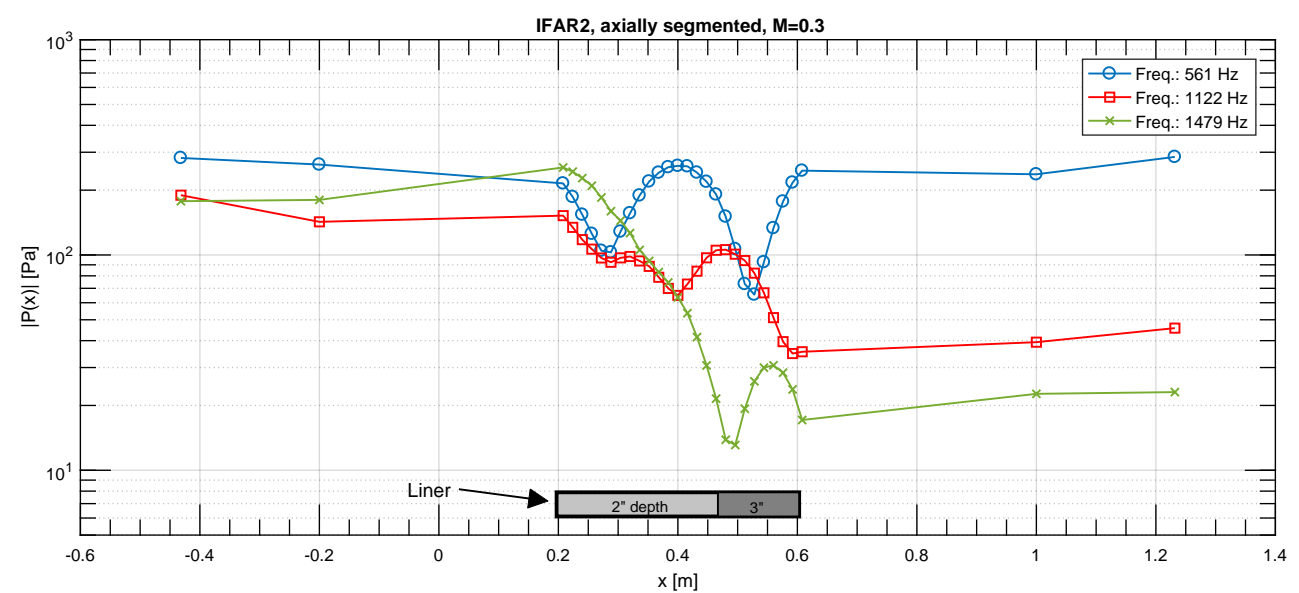

Figure 9. Axial pressure profiles with IFAR2 liner sample at centerline Mach number 0.3 for three different frequencies: $714 \mathrm{~Hz}$ (blue line; low dissipation), $916 \mathbf{H z}$ (red line; first dissipation maximum) and $1326 \mathrm{~Hz}$ (red line; second dissipation maximum).

a frequency with a low dissipation coefficient, here $561 \mathrm{~Hz}$ (blue line), is again mainly dominated by a standing wave pattern with little reduction in the sound pressure amplitude along the duct axis $(x)$.

\section{IV.C. Wave Number Determination}

The next step towards the impedance eduction is the determination of the axial wave numbers of all existing modes in the lined section. Here, a combination of two different methods (Kumaresan and Tufts method (KT) and Matrix Pencil method (MP)) combined with a manual sorting procedure was applied. The KT and MP methods are explained in detail in Weng et al. ${ }^{4,12,13}$ and are only briefly introduced here:

The KT method is a Prony-like method. It detects the system poles of the acoustic wave field description by finding the roots of a linear prediction polynomial. The corresponding linear system of equations of the polynomial coefficients is solved in advance via a singular value decomposition (SVD). In order to select only the duct-moderelated singular values and to exclude noise-related contributions the SVD is truncated by a criterion based on the minimum description length (MDL). ${ }^{14}$

While the KT method finds the system poles in two steps (solving the linear system of prediction equations and finding the roots of the prediction polynomial), the MP method finds the system poles and therewith the duct mode wave numbers directly by solving a generalized eigenvalue problem, called "matrix pencil". The MP methods involves a certain prefiltering of the noise-contaminated data by performing also a truncated SVN (similar to the KT method). Here, again the MDL approach is used to estimate the relevant duct-mode-related singular values and reject or reduce noise influences. Compared to the KT method the MP method is able to identify the modes propagating in both axial duct directions (downstream: $x^{+}$and upstream: $x^{-}$) at the same time. Previous investigations on the performance 

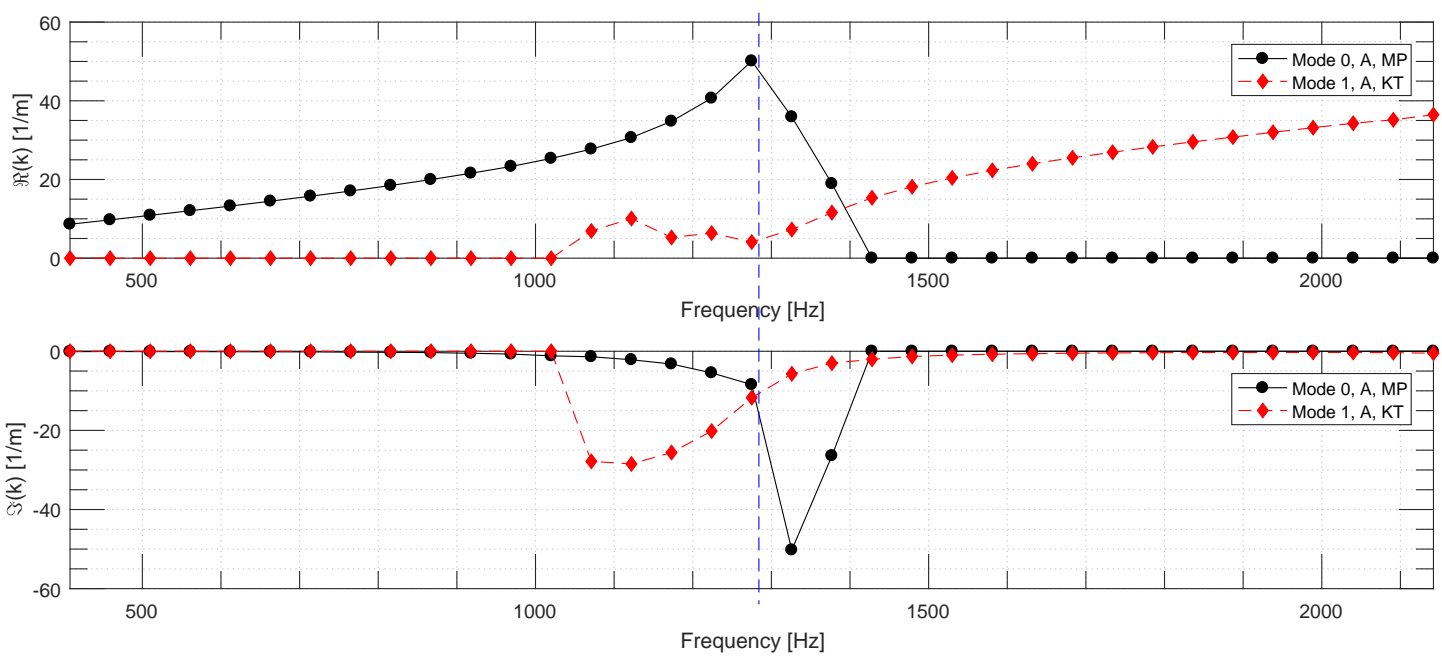

Figure 10. Real part (above) and imaginary part (below) of the determined wave numbers for IFAR1 liner sample in the no grazing flow case with mode crossing between $1275 \mathrm{~Hz}$ and $1326 \mathrm{~Hz}$ (dashed blue line).

of these two wave number determination methods ${ }^{12}$ revealed that it may be beneficial to apply both methods together in practice since both methods behave different at low signal-to-noise ratios which allows a manual selection of the resulting wave numbers based on a plausibility assessment.

The careful determination of the dominating wave numbers becomes exceptional important when a so called mode crossing appears in the lined section. Mode crossing denotes the change of the least attenuated mode from the fundamental mode ("plane wave") to a next higher order mode above a certain frequency. This higher order mode is then less damped than the fundamental mode. This effect was observed especially at liner samples with a low resistance value and earlier described for example in detail by Schulz et al. ${ }^{1 .}$

Here, this mode crossing also appears in the no flow and low Mach number flow cases for the IFAR1 and IFAR2 liner samples due to their fairly high facesheet porosity (about 12\%) and the corresponding low wall resistance. Figure 10 shows the wave numbers for the IFAR1 sample without grazing flow (above: real part; below: imaginary part) with an excitation from upstream (speaker A). Above a frequency of about $1300 \mathrm{~Hz}$ (dashed blue line) the absolute value of the imaginary part (describing the damping of the wave) of the fundamental mode (mode 0; black line) exceeds the corresponding value of the next higher order mode (mode 1; red line). This indicates that above this frequency the dominating, energy transporting duct mode changes to this higher order mode which should be accordingly selected for the impedance eduction procedure.

\section{IV.D. Liner Impedance}

In general the test rig setup and the test procedure at the DUCT-R allows different techniques, modal based and nonmodal based, to educe the impedance. A comparative study of these methods was presented in earlier work. ${ }^{4}$ In the framework of this IFAR liner benchmark the primary focus was set on the modal based techniques applying the determined wave numbers as described above.

Following the description of the acoustic pressure field for each mode by $\tilde{p}=\hat{p}(y) e^{-i k x}$, with the axial wave number of the respective mode $k$, the Convected Helmholtz Equation (CHE) reduces to a one-dimensional form:

$$
\frac{\mathrm{d}^{2} \hat{p}}{\mathrm{~d} y^{2}}+k_{y}^{2} \hat{p}=0
$$

where $k_{y}^{2}=k_{0}^{2}\left[\left(1-M_{\mathrm{avg}} \Gamma\right)^{2}-\Gamma^{2}\right]$ is the wall-normal wave number squared, $k_{0}=\omega / c_{0}$ is the free-space wave number, $\omega$ is the angular frequency, $c_{0}$ is the reference speed of sound, $\Gamma=k_{x} / k_{0}$ is the dimensionless axial wave number and $M_{\text {avg }}$ is the Mach number averaged over the duct cross section. With the Ingard-Myers condition ${ }^{16,17}$ at $y=0$ (liner surface) and the hard-wall boundary condition at $y=H$, the following nonlinear dispersion relation can be derived ${ }^{18}$ from equation 11 :

$$
\zeta=\frac{\mathrm{i} k_{0}\left(1-M_{\mathrm{avg}} \Gamma\right)^{2}}{k_{y} \tan \left(k_{y} H\right)} .
$$


Once the wave number $\Gamma$ of a mode is determined following the procedure described in section IV.C, the normalized liner impedance $\zeta$ can be calculated from equation 12. Since the calculation is purely analytic and iteration-free, this eduction method is referred to as the straightforward method by Jing et al. ${ }^{18}$.
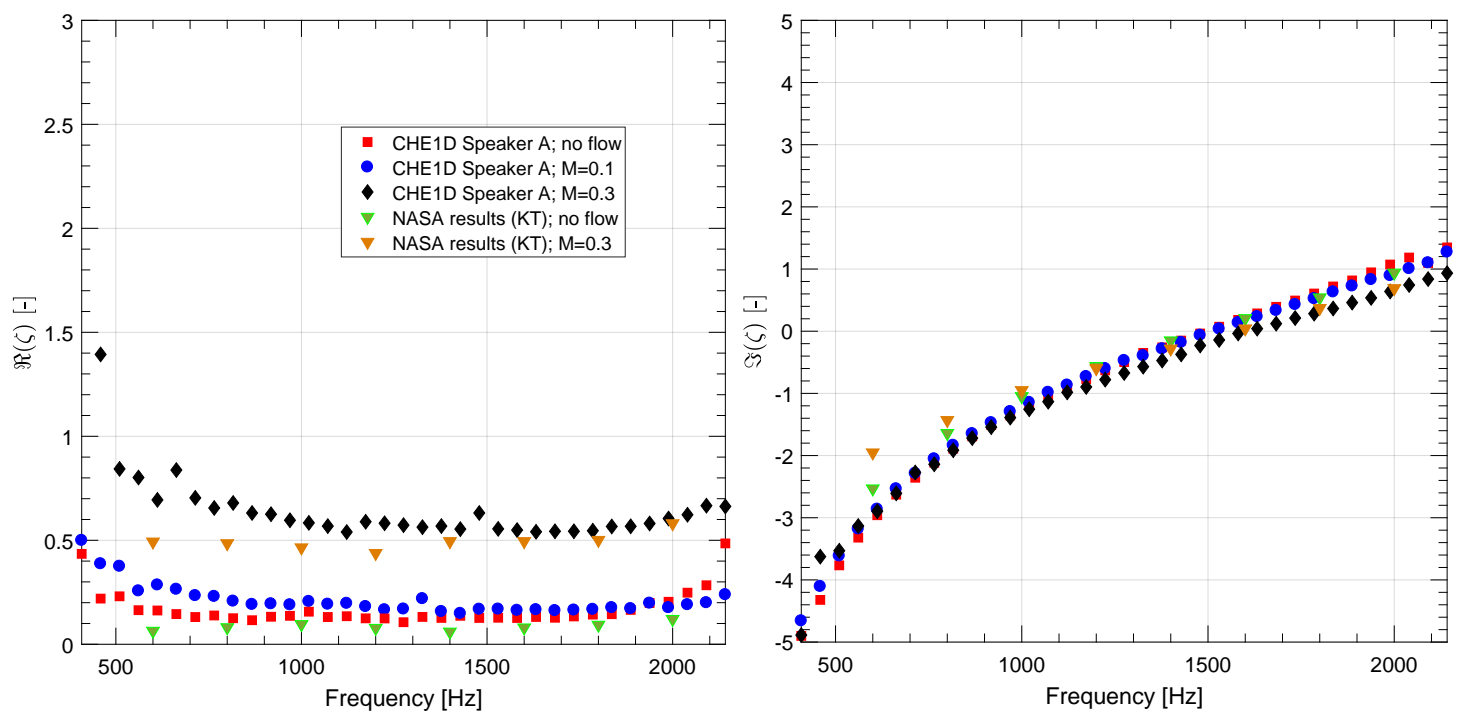

Figure 11. Real part (resistance; left) and imaginary part (reactance; right) of the educed liner impedance for the IFAR1 liner sample for three flow cases (no flow: red squares; $M=0.1$ : blue circles; $M=0.3$ : black diamonds). NASA results: ${ }^{1}$ no flow: green triangles; $M=0.3:$ orange triangles.

The impedance results for the IFAR1 liner sample are shown in figure 11 (left: resistance $\Re(\zeta)$; right: reactance $\Im(\zeta)$ ). Without grazing flow (red squares) the resistance is fairly low $(\approx 0.2)$ and nearly constant over the measured frequency range $(400 \mathrm{~Hz}-2100 \mathrm{~Hz})$. The reactance shows a resonator typical curve progression from negative values to positive values crossing the zero at the resonance frequency of about $1500 \mathrm{~Hz}$. Under low grazing flow conditions with a centerline Mach number of $M=0.1$ (blue circles) the resistance slightly increases while the reactance does not change very much. With a grazing flow Mach number of $M=0.3$ (black diamonds) the resistance increases as expected up to values between 0.5 and 0.7 . Here, the reactance shows a shift of the resonance frequency to $\approx 1600 \mathrm{~Hz}$.
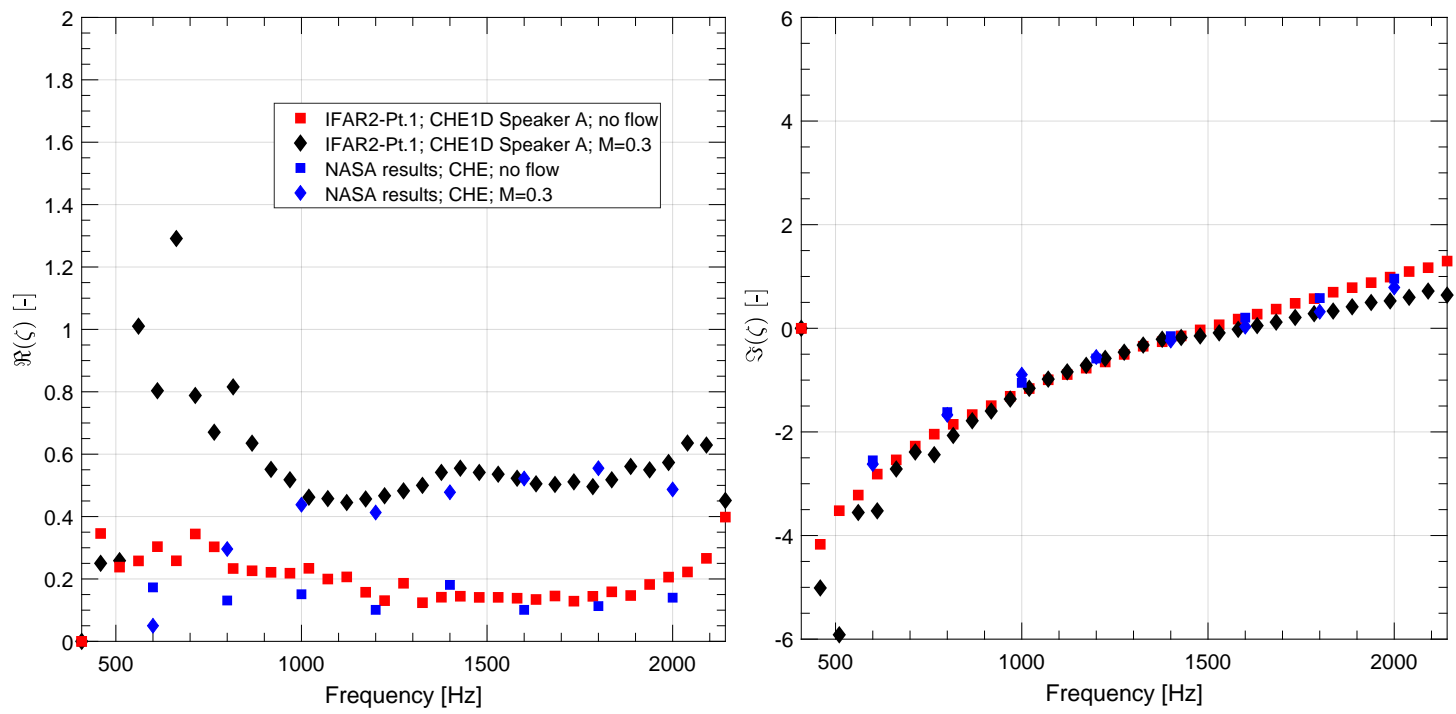

Figure 12. Real part (resistance; left) and imaginary part (reactance; right) of the educed liner impedance for the first part of the IFAR2 liner sample (IFAR2Pt.1; $2^{\prime \prime}$ cavity depth) for two flow cases (no flow: red squares, $M=0.3$ : black diamonds) compared to the NASA results ${ }^{1}$ (no flow: blue squares, $M=0.3$ : blue diamonds).

The corresponding values from the NASA GFIT test rig ${ }^{1}$ are also plotted in figure 11 for the no flow case (green triangles) and for the case with a centerline Mach number of $M=0.3$ (orange triangles). It should be noted that 
even with the same measured centerline Mach number the average Mach number in the duct is probably different between the GFIT test rig and the DUCT-R due to different cross-sectional dimensions and hence, different flow profiles. However, the comparison of the resistance (figure 11; left) and reactance (figure 11; right) data show a very close match between DLR and NASA results. Only for lower frequencies below around $800 \mathrm{~Hz}$ some deviations (in the order of $30 \%$ for the reactance and the resistance with flow Mach number of 0.3) can be observed.
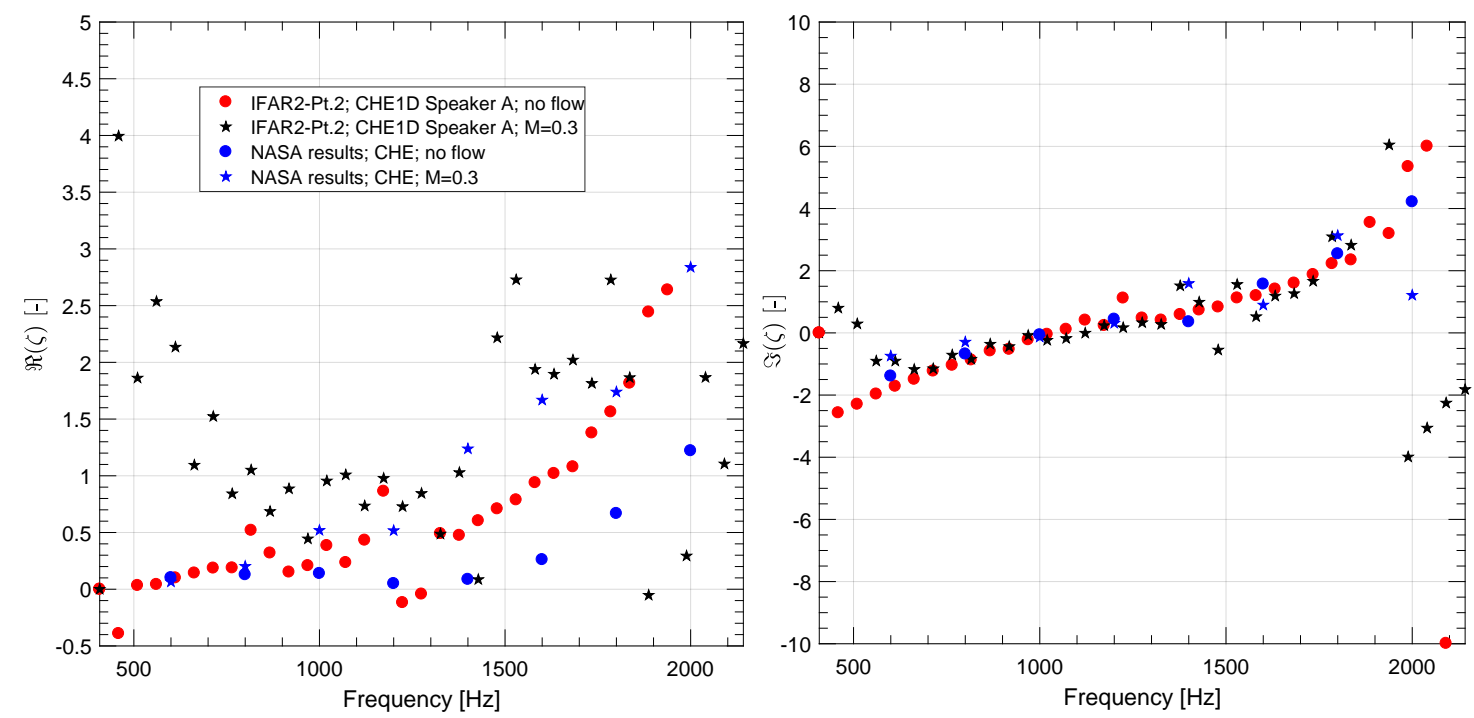

Figure 13. Real part (resistance; left) and imaginary part (reactance; right) of the educed liner impedance for the second part of the IFAR2 liner sample (IFAR2-Pt.2; $3^{\prime \prime}$ cavity depth) for two flow cases (no flow: red circles, $M=0.3$ : black stars) compared to the NASA results ${ }^{1}$ (no flow: blue circles, $M=0.3$ : blue stars).

For the IFAR2 liner samples the wave number determination described in section IV.C has been applied for the two axial sections of the liner sample (with different cavity depths) by selecting the appropriate face-to-face microphones in each section. The lower number of microphones used for the impedance evaluation of each section causes a higher scattering and uncertainty of the wave number values especially for the second liner segment where only 5 microphones for the no flow case and 8 microphones for the Mach 0.3 case could be employed. A sensitivity study (not shown here) revealed that relatively small errors in the wave number in the order of few percent can have a high impact on the uncertainty of the impedance results at certain frequencies. This is caused by the non-linear relationship given by equation 12 which magnifies measurement errors of the wave number at frequencies with a greater distance to the resonance frequency of the liner.

Figure 12 displays the impedance values for the first liner segment of IFAR2 (IFAR2-Pt.1; 2" cavity depth) for two flow conditions (no flow: red squares; $M=0.3$ : black diamonds). The corresponding NASA results ${ }^{1}$ are added $^{2}$ in blue symbols (no flow: blue squares; $M=0.3$ : blue diamonds). With respect to the resistance (figure 12; left) the DLR values match very well the NASA data. The DLR data exhibit with grazing flow (black diamonds) only minor scattered resistance results for low frequencies below $700 \mathrm{~Hz}$ (similar to the NASA data). With grazing flow ( $M=0.3$ ) there is some systematic deviation in the reactance (figure 12; right) between DLR (black diamonds) and NASA (blue diamonds) observable for low frequencies below $1100 \mathrm{~Hz}$. This behaviour is not fully understood yet.

The impedance for the second part (IFAR2-Pt.2; $3^{\prime \prime}$ cavity depth) in figure 13 indicates stronger scattered values for low frequencies $<700 \mathrm{~Hz}$ and above $\approx 1500 \mathrm{~Hz}-1700 \mathrm{~Hz}$ especially for the grazing flow case for both the DLR (black stars) as well as the NASA (blue stars) data. This is presumably caused by the decreased signal-to-noise ratio with grazing flow. However, the reactance zero crossing and therewith the liner resonance frequency is identified reasonably accurate to $\approx 1050 \mathrm{~Hz}$ for the IFAR2-Pt.2 ( $3^{\prime \prime}$ cavity depth; no flow) segment.

Comparing the results from the constant depth sample IFAR1 ( $2^{\prime \prime}$ cavity depth) to the IFAR2-Pt.1 data (also $2^{\prime \prime}$ cavity depth) in figure 14 shows a close match between IFAR1 (filled symbols) and the IFAR2-Pt.1 (open symbols). Only for the low frequency range below $800 \mathrm{~Hz}$ the results for the IFAR2-Pt.1 segment show some more scattered impedance values. This is caused by the shorter length which reduces the number of face-to-face microphones and therewith increases the uncertainty of the wave number determination (KT/MP). 

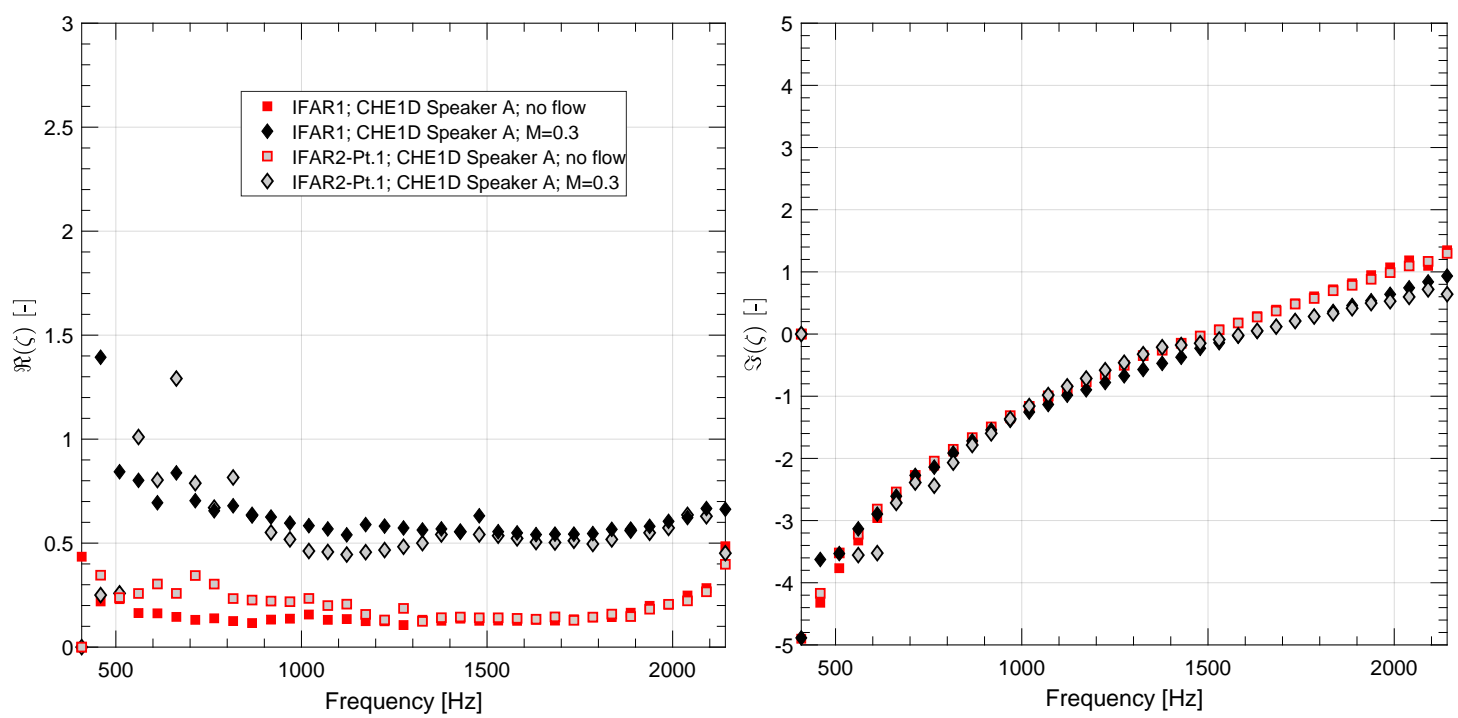

Figure 14. Real part (resistance; left) and imaginary part (reactance; right) of the educed liner impedance for the IFAR1 liner sample ( $2^{\prime \prime}$ cavity depth) and the first part of the IFAR2 liner sample (also $2^{\prime \prime}$ cavity depth) for two flow cases. IFAR1: no flow: filled red squares, $M=0.3:$ filled black diamonds; IFAR2 part 1: no flow: open red squares, $M=0.3$ : open black diamonds.

\section{Conclusions and Outlook}

The DLR contribution to the IFAR liner benchmark challenge \#1 was presented here. Starting with the additive manufacturing and the test rig integration of two different liner samples - one with constant cavity depth (IFAR1) and one axially segmented with two different cavity depths (IFAR2) - the entire processing and acoustic characterization can now be compared to other partner's contribution within the framework of this benchmark.

The IFAR1 liner sample shows a dissipation behaviour typical for resonator liners with a distinct maximum damping frequency around $1300 \mathrm{~Hz}$. In contrast the IFAR2 sample reveals two dissipation maxima related to the quarterwavelength resonance frequencies of each liner segment, respectively.

The corresponding axial pressure profiles confirm this observation and provide some insight into the total sound pressure level at each microphone position for the different configurations. One key processing step for the impedance eduction is the determination of the axial wave numbers of the dominating modes in the lined sections. Hereby, a combination of two methods (KT/MP) with a specific truncation algorithm (MDL criterion) was applied to the data of the face-to-face microphones above the lined wall. A crucial processing component is here the manual mode selection based on plausibility assessment.

While different, modal based and non-modal based impedance eduction techniques are available at DLR, for the sake of brevity only the results of the so-called straightforward method, based on an analytic solution of the onedimensional Convected Helmholtz Equation (CHE1D), were presented here. In this framework also another modal based impedance eduction method by solving the Pridmore-Brown equation over the full duct cross-section (PBECroSec) was applied. And although the PBE-CroSec takes into account visco-thermal losses as well as shear flow effects, here this method yield fairly similar results like the CHE1D.

The impedance eduction for the constant depth liner sample IFAR1 shows reasonable results for the different grazing flow cases. More challenging appears the wave number determination for the two different axial parts of the segmented liner IFAR2. One main reason here is the reduced number of microphones which are e.g. for the second part of IFAR2 only 5-8 microphones (compared to 19-20 microphones for IFAR1). The strong scattering behaviour within the impedance results can be explained by the strong non-linear relation of the impedance and the axial wave number in equation 12. Small uncertainties in the wave number values result in a larger uncertainty error of the educed impedance data. This applies especially for the shorter segment IFAR2-Pt.2. Due to an decreased signal-to-noise ratio this effect is even further augmented under grazing flow conditions.

The comparison to the NASA impedance results ${ }^{1}$ shows a very good agreement. The overall evaluation demonstrates the liner impedance eduction capability even for complex non-homogeneous liner samples and the results can be compared to the findings of other IFAR liner benchmark participants. 


\section{Acknowledgments}

The authors highly appreciate the support of Wolfram Hage and Sebastian Kruck in carefully manufacturing and post processing the liner samples. The authors also like to acknowledge the effort of Michael G. Jones from NASA Langley Research Center in organizing and advising this IFAR liner benchmark.

\section{References}

${ }^{1}$ Jones, M., Watson, W., Nark, D., and Howerton, B., "Impedance Eduction for Multisegment Liners," 24rd AIAA/CEAS Aeroacoustics Conference, No. 2018-3441, Atlanta, Georgia, USA, June 2018.

${ }^{2}$ Busse-Gerstengarbe, S., Bake, F., Enghardt, L., and Jones, M. G., "Comparative Study of Impedance Eduction Methods, Part 1: DLR Tests and Methodology," 19th AIAA/CEAS Aeroacoustics Conference, No. 2013-2124, Berlin, Germany, May 2013.

${ }^{3}$ Schulz, A., Weng, C., Bake, F., Enghardt, L., and Ronneberger, D., "Modeling of liner impedance with grazing shear flow using a new momentum transfer boundary condition," 23rd AIAA/CEAS Aeroacoustics Conference, No. 2017-3377, Denver, Colorado, USA, June 2017.

${ }^{4}$ Weng, C., Enghardt, L., and Bake, F., "Comparison of non-modal-based and modal-based impedance eduction techniques," 24th AIAA/CEAS Aeroacoustics Conference, No. 2018-3773, Atlanta, Georgia, USA, June 2018.

${ }^{5}$ Lahiri, C., Sadig, S., Gerendas, M., Enghardt, L., and Bake, F., "Establishment Of A High Quality Database For The Modelling Of Perforated Liners," Journal of Engineering for Gas Turbines and Power, Vol. 133, No. 9, September 2011, pp. 091503-1 - 091503-9.

${ }^{6}$ Ronneberger, D., Genaue Messung der Schalldämpfung und der Phasengeschwindigkeit in durchströmten Rohren im Hinblick auf die Wechselwirkung zwischen Schall und Turbulenz, Habilitation thesis, Universität Göttingen, 1975.

${ }^{7}$ Brandes, M., Optimierung eines Meßvervahrens zur Bestimmung von akustischen Parametern im durchströmten Rohr, Diploma thesis, Universität Göttingen, 1992.

${ }^{8}$ Enghardt, L., Bestimmung akustischer Streufaktoren im durchströmten Rohr-Optimierung von Mikrofonanordnung und Meï氵œprozedur, Diplomarbeit, Universität Göttingen, Göttingen, 1992.

${ }^{9}$ Chung, J. Y., "Rejection of flow noise using coherence function method," Journal of the Acoustical Society of America, Vol. 62, 1977, pp. $388-395$.

${ }^{10}$ Blokhintsev, D. I., Acoustics of a nonhomogeneous moving medium, NACA Technical Memorandum 1399, 1956, Originally published 1946 in russian language.

${ }^{11}$ Morfey, C. L., "Acoustic energy in non-uniform flows," Journal of Sound and Vibration, Vol. 14, No. 2, 1971, pp. $159-170$.

${ }^{12}$ Weng, C., Schulz, A., Ronneberger, D., Enghardt, L., and Bake, F., "Impedance eduction in the presence of turbulent shear flow using the linearized Navier-Stokes equations," 23rd AIAA/CEAS Aeroacoustics Conference, No. 2017-3182, Denver, Colorado, USA, June 2017.

${ }^{13}$ Weng, C., Schulz, A., Ronneberger, D., Enghardt, L., and Bake, F., "Flow and Viscous Effects on Impedance Eduction," AIAA Journal, Vol. 56, No. 3, 2018, pp. $1118-1132$.

${ }^{14}$ Wax, M. and Kailath, T., "Detection of signals by information theoretic criteria," IEEE Transactions on Acoustics, Speech, and Signal Processing, Vol. 33, No. 2, Apr 1985, pp. 387-392.

${ }^{15}$ Schulz, A., Bake, F., Enghardt, L., and Ronneberger, D., "Impedance Eduction of Acoustic Liners Based on Four Different Levels of Physical Modeling," 22nd AIAA/CEAS Aeroacoustics Conference, No. 2016-2726, Lyon, France, May/June 2016, Number of citations (Google Scholar 2018): 2.

${ }^{16}$ Ingard, U., "Influence of Fluid Motion Past a Plane Boundary on Sound Reflection, Absorption, and Transmission," The Journal of the Acoustical Society of America, Vol. 31, No. 7, 1959, pp. 1035-1036.

${ }^{17}$ Myers, M. K., "On the acoustic boundary condition in the presence of flow," Journal of Sound and Vibration, Vol. 71, No. 3, 1980, pp. 429434.

${ }^{18}$ Jing, X., Peng, S., and Sun, X., "A straightforward method for wall impedance eduction in a flow duct," The Journal of the Acoustical Society of America, Vol. 124, No. 1, 2008, pp. 227-234. 